\title{
ECG Analysis Based On Window Filtering Approach Using Empirical Mode Decomposition Technique
}

\author{
Rovin Tiwari ${ }^{1}$, Prof. Rahul Dubey ${ }^{2}$, Prof. N.K. Mittal ${ }^{3}$ \\ ${ }^{1,2,3}$ Department of Electronics and communication, OIST, Bhopal
}

\begin{abstract}
Electrocardiogram (ECG) signal is a very important measure to know the Heart actual conditions. It becomes necessary to make ECG signals free from noise for proper analysis and detection of the diseases. In this paper a new approach based on the window filtering using Empirical Mode Decomposition technique is presented. EMD is a relatively new, data driven adaptive technique used to decompose ECG signal into a series of Intrinsic Mode Functions (IMFs). Different ECG signals are used to verify the proposed method using $M A T L A B$ software. Method presented in this paper is based on rectangular and Kaiser Window filtering method to ECG signal and calculate mean square value using Hard and soft Thresholding. For analyses purpose, extensive simulations are carried out using the MIT-BIH database and the performances are evaluated in terms of standard metrics namely, Features of signal, SNR improvement in dB, Mean Square Error (MSE) and Percent Root Mean Square Difference (PRD).
\end{abstract}

Keywords : ECG, Window filtering, Rectangular, Kaiser, EMD, CSE and MIT-BIH database.

\section{Introduction}

An electrocardiogram is used to monitor your heart. Each beat of our heart is triggered by an electrical impulse normally generated from special cells in the upper right chamber of your heart. An electrocardiogram — also called an ECG or EKG shows in figure1.[11]

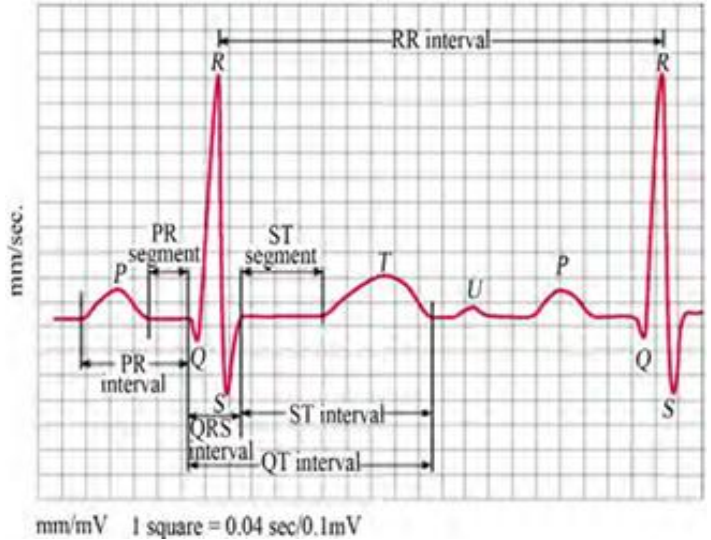

Figure1.Different features in ECG signal

Automatic classification ECG signal consist of different features of ECG in one cardiac cycle. Features relating to fiducial point intervals were considered for each heart beat Features relating to heartbeat intervals and ECG morphology were also calculated separately for each heartbeat in the ECG signals.

The RR-interval is the time between successive RR-peaks, the inverse of this time interval gives the instantaneous heart rate. A series of RR-intervals is known as a RR tachogram and variability of these RRintervals reveals important information about the physiological state of the subject. In this paper we generate a synthetic ECG signal with realistic PQRSTU morphology. SNR is the powerful parameter to decide the quality and analysis of signal. By using this proposed method the SNR is obtained highest. This paper deals with the study of FIR filtering of ECG signals. The performance of FIR filter is evaluated on several ECGs recordings, and studying the SNR and morphology of the filter outputs. We find FIR filter with rectangular and Kaiser Window works excellent [2].

EMD is intuitive and adaptive, with basic functions derived fully from the data.. The key task here is to identify the intrinsic oscillatory modes by their characteristic time scales in the signal empirically, and accordingly, decompose the signal into intrinsic mode functions (IMFs) [1]. A function is considered to be an IMF if it satisfies two conditions [9]; First, In the whole data set, the number of local extrema and that of zero crossings must be equal to each other or different by at most one and second, at any point, the mean value of the envelope defined by the local maxima and that defined by the local minima should be zero. Windowing of a 
simple waveform like cos $\omega$ t causes its Fourier transform to develop non-zero values (commonly called spectral leakage) at frequencies other than $\omega$. The leakage tends to be worst (highest) near $\omega$ and least at frequencies farthest from $\omega$. The rectangular and Kaiser window has excellent resolution characteristics for sinusoids of comparable strength.

Wavelets have been found to be a powerful tool for analysis from a variety of signals. Today signal processing plays a major role in ECG signal analysis and interpretation. Wavelets are mathematical functions that cut up data into different frequency components, and then study each component with a resolution matched to its scale. We are using hard thresholding and soft thresholding for calculate mean square value[4].

\section{Dataset}

The dataset used in this study is obtained from CSE and "MIT-BIH Arrhythmia Database"[12] available on-line. The source of the ECGs included in the MIT-BIH Arrhythmia Database is a set of over 4000 long- term Holter recordings that were obtained by the Beth Israel Hospital Arrhythmia Laboratory between 1975 and 1979. Approximately $60 \%$ of these recordings were obtained from inpatients [12].

\section{Proposed Methodology}

Here we are showing flow chart in following figure.

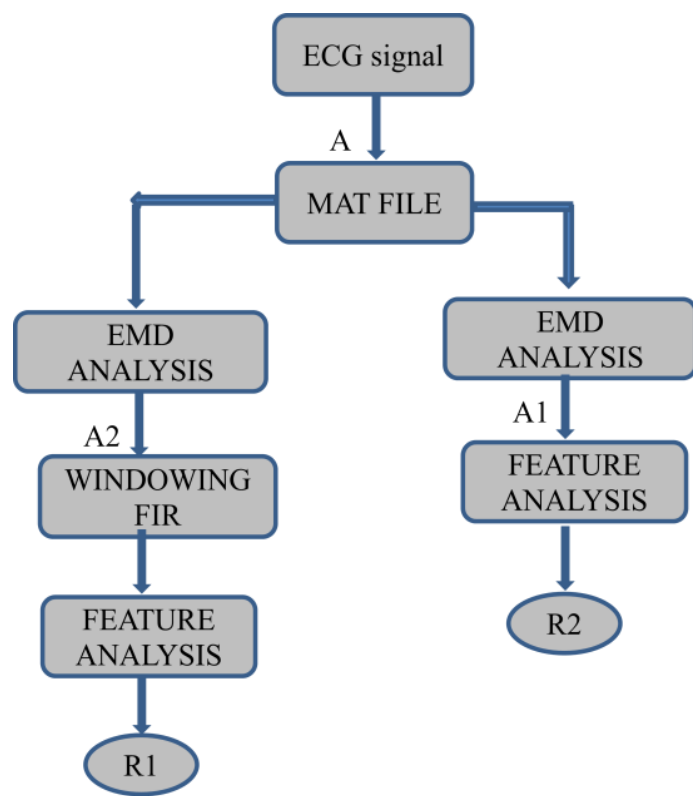

Figure2. Flow chart of proposed algorithm

\section{Empirical Mode Decomposition (EMD)}

EMD can decompose ECG signal into a series of IMFs subjected to the following steps [7]:

1. Finding all the local extrema, including maxima and minima; then connecting all the maxima and minima of signal $x(t)$ using smooth cubic splines to get its upper envelope $x u p(t)$ and lower envelope xlow $(t)$.

2. Subtracting mean of these two envelopes $m 1(t)=(x u p(t)+x l o w(t)) / 2$ from the signal to get their difference:

$$
\mathrm{h} 1(\mathrm{t})=\mathrm{x}(\mathrm{t})-\mathrm{m} 1(\mathrm{t})
$$

3. Regarding the h1(t) as the new data and repeating steps 1 and 2 until the resulting signal meets the two criteria of an IMF, defined as c1(t). The first IMF c1(t) contains the highest frequency component of the signal. The residual signal $\mathrm{r} 1(\mathrm{t})$ is given by :

$$
\mathrm{r} 1(\mathrm{t})=\mathrm{x}(\mathrm{t})-\mathrm{c} 1(\mathrm{t})
$$

4. Regarding r1(t) as new data and repeating steps (1) (2) (3) until extracting all the IMFs. The sifting procedure is terminated until the Mth residue $\mathrm{rM}(\mathrm{t})$ becomes less than a predetermined small number or becomes monotonic.

\section{Window Filtering approach}

Window techniques are commonly used for digital filter design such as Finite duration unit pulse response (FIR) filter. FIR filter is one of the techniques successfully employed in processing ECG signals for measurement and noise reduction[6]. They are used to modify the bio-signal by removing noise and the effect of a system on the input signal can be described in both time and frequency domains. The time domain method of 
ECG signal analysis is not always sufficient to study all the features of ECG signals. Therefore, the frequency representation of a signal is required. Hence, filters are designed in frequency domain [3]. Here, we are using rectangular window and Kaiser Window for ECG signal.

\subsection{Rectangular window}

The rectangular window is the simplest window, equivalent to replacing all but $N$ values of a data sequence by zeros, making it appear as though the waveform suddenly turns on and off: $w(n)=1$.

Other windows are designed to moderate these sudden changes because discontinuities have undesirable effects on the discrete-time Fourier transform (DTFT) and/or the algorithms that produce samples of the DTFT. The rectangular window is the 1st order B-spline window as well as the 0th power cosine window [6].

The rectangular function is given by-

$\mathrm{W}_{\mathrm{R}}(\mathrm{n})=\left\{\begin{array}{c}1, \text { for }|n| \leq \frac{M-1}{2} \\ 0, \text { otherwise }\end{array}\right.$

Where $\mathrm{N}$ represents the width, in samples, of a discrete-time, symmetrical window function $\mathrm{w}[\mathrm{n}], 0 \leq \mathrm{n}$ $\leq \mathrm{N}-1$. When $\mathrm{N}$ is an odd number, the non-flat windows have a singular maximum point. When $\mathrm{N}$ is even, they have a double maximum. $\mathrm{M}$ is rectangular window length [3].

\subsection{Kaiser window}

Kaiser based FIR filter is designed to remove the unwanted frequency component which corresponds to the noise. Kaiser window has very desirable characteristics both in time domain and frequency domain. A good window should be a time limited function with a Fourier transform that is band limited and Kaiser Window possesses such characteristics [2]

To obtain a Kaiser window that designs an FIR filter with side lobe attenuation of $\alpha \mathrm{dB}$, Kaiser window parameter $\beta$ that affects the side lobe attenuation of the Fourier transform of the window is given by

$$
\beta=\left\{\begin{array}{l}
0.1102(\alpha-8.7), \alpha>50 \\
0.582(\alpha-21)^{0.4}+0.07886(\alpha-21), 21 \leq \alpha \leq 50 . \\
0, \alpha<21
\end{array}\right.
$$

$\mathrm{N}=(\alpha-8 / 2.285 \Delta \mathrm{w})+1$

Here $\mathrm{N}$ is the filter order,$\Delta \mathrm{w}$ is the width of the smallest transition region and $\alpha=-20 \log 10 \delta$ is the stop band attenuation expressed in decibels. Increasing $\beta$ widens the main lobe and decreases the amplitude of the side lobes (i.e., increases the attenuation)[3].

\section{Wavelet Thresholding}

In wavelet transform, a signal is analyzed and expressed as a linear combination of the sum of the product of the wavelet coefficients and mother wavelet. The filtering operations in DWT result in a change in the signal resolution [5], whereas sub sampling (down sampling/up sampling) causes change of the scale. Thus, DWT gives detail information thereby helping in analyzing it at different frequency bands with different resolutions.

A general equation for the DWT transformed signal is written as [11],

$$
X\lfloor a, b\rfloor=\sum_{n=-\infty}^{\infty} x\lfloor n\rfloor \phi_{a, b}\lfloor n\rfloor
$$

where, $x[n]$ is the given ECG signal to be transformed and

$$
\phi_{a, b}[n]=\left(\frac{1}{\sqrt{a}}\right) \times \phi\left[\frac{n-b}{a}\right]
$$

In DWT, the function $\varphi[\mathrm{n}]$ represents a window of finite length, where, $\mathrm{b}$ is a real number known as window translation parameter and a is a positive real number named as dilation or contraction parameter

The idea of thresholding is to set to zero all coefficients that are less than a particular threshold. In this paper, we have evaluated the performance of the following threshold estimators on the analysis of ECG signal [9]. 


\subsection{Hard thresholding}

Hard Thresholding: The hard thresholding operation is defined as , $D(U, \lambda)=U$ for all $|U|>\lambda$.and $D(U$, $\lambda)=0$ for all $|\mathrm{U}|<\lambda$ Hard thresholding in the DWT domain zeroes out all the signal values smaller than $\delta$. As a result there appears discontinuity in the reconstructed signal. In equation form hard thresholding operation performed on a particular detailed DWT coefficient di on a wavelet level 1 can be expressed as[5],

$$
\tilde{X}_{d i}(l)=\left\{\begin{array}{cc}
\left|\tilde{X}_{d i}(l)\right|, & \left|\tilde{X}_{d i}(l)\right| \geq \delta_{l} \\
0, & \left|\tilde{X}_{d i}(l)\right|<\delta_{l},
\end{array}\right.
$$

Where, $\mathrm{i}$ stand for the index of the detailed DWT coefficients at a level 1. usually hard thresholding is used for data compression purpose.

\subsection{Soft thresholding}

Soft Thresholding: The soft thresholding operation is defined as, $D(U, \lambda)=\operatorname{sgn}(U) \max (0,|U|-\lambda)$. In soft thresholding, signal values smaller than $\delta \square$ are turned to zero and $\delta \square$ is subtracted from the signal values greater than In equation form the thresholding operation performed on a particular detailed DWT coefficient $i d$ on a wavelet level $l$ can be expressed as[10],

$$
\tilde{X}_{d i}(l)=\left\{\begin{array}{cc}
\left|\tilde{X}_{d i}(l)\right|-\delta_{l}, & \left|\tilde{X}_{d i}(l)\right| \geq \delta_{l} \\
0, & \left|\tilde{X}_{d i}(l)\right|<\delta_{l},
\end{array}\right.
$$

\section{Parameter Selection}

SNR is an important parameter while evaluation or processing of any signal and it given by the following equation, SRN[12]

$$
=10 \log _{10}\left[\frac{\sum_{\mathrm{i}=1}^{\mathrm{N}}(\text { filtered signal })^{2}}{\sum_{\mathrm{i}=1}^{\mathrm{N}}(\text { original signal }- \text { filtered signal })^{2}}\right]
$$

Let $x[n]$ and $x[n]$ be the original and the reconstructed signals, respectively, and $N$ its length. The PRD formula is defined as:

$$
\mathrm{PRD}=\sqrt{\frac{\sum_{n=1}^{N}(x[n]-\hat{x}[n])^{2}}{\sum_{n=1}^{N}(x[n])^{2}}} \times 100
$$

\section{Simulations And Results}

Testing of ECG signal was performed on artificially noised signals from the standard CSE and MITBIH database. Results show that analysis schemes involving window, EMD and wavelet domains are more accurately.

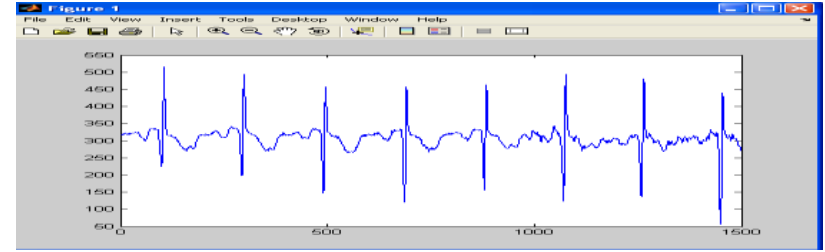

(a)

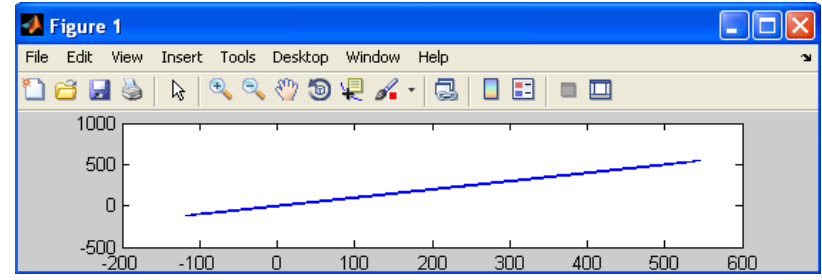

(b)

Figure 3: (a) ECG signal (b) signal characteristic 
For the simulation of the proposed method, an ECG signal has been taken as original signal, shown in Fig. 3(a). The sampling frequency is $800 \mathrm{~Hz}$ and 2048 samples of the signal are used. Characteristic of ECG signal shown in Fig. 3(b), both are used as the test signal for the simulation of the proposed method.

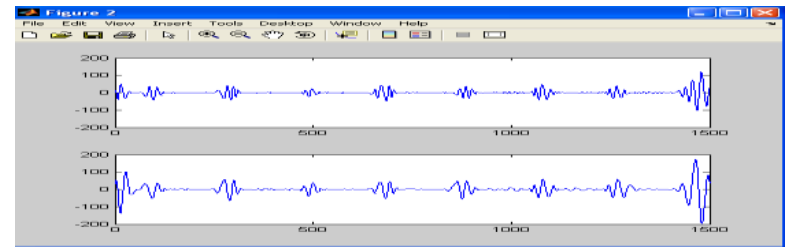

(a)

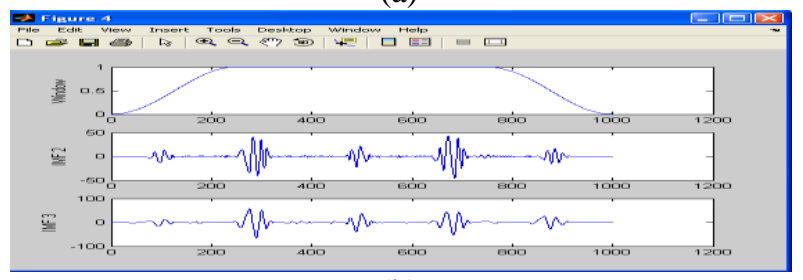

(b)

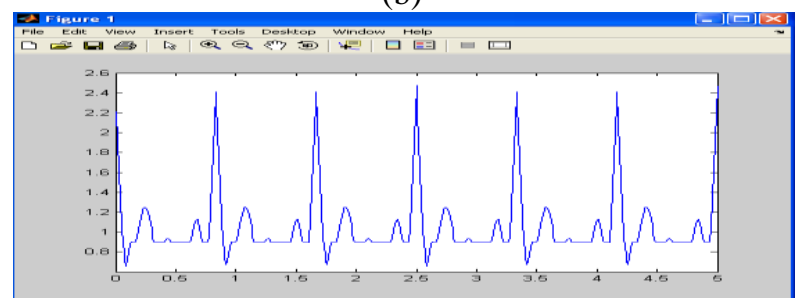

(c)

Figure 4: (a) EMD-IMF (b) Windowing and EMD (IMF-I and IMF-II) (c) ECG signal after Thresholding

The wavelet hard and soft thresholding and EMD were used in all the experiments. Fig. 4(a) shows the intrinsic mode function of EMD, Fig. 4(b) shows the windowing results and Fig. 4(c) shows the denoised ECG signal results. The simulation is done using MATLAB 11 To evaluate the performance of the proposed method PRD is computed \& also signal to noise ratio (SNR) is measured. The results obtained, from the simulation, are given in the Table 1 and Table 2.

Table 1: Results of ECG Signals Denoised using proposed method

\begin{tabular}{|l|l|l|}
\hline \multicolumn{1}{|c|}{ SNR } & \multicolumn{1}{|c|}{ PRD } & \multicolumn{1}{c|}{ Signals } \\
\hline 6.3674 & 0.4393 & Signal 1 \\
\hline 7.9527 & 2.6114 & Signal 2 \\
\hline 8.5999 & 1.3985 & Signal 3 \\
\hline 9.0747 & 1.0207 & Signal 4 \\
\hline
\end{tabular}

Table2: Data list of ECG simulation

\begin{tabular}{|c|c|c|c|}
\hline \multicolumn{3}{|c|}{ ECG data } \\
\hline Heartbeat rate & $\mathbf{a}$ & $\mathbf{u 1}$ & $\mathbf{u 2}$ \\
\hline 65 & 0.035 & 2.1667 & 0 \\
\hline 70 & 0.035 & 2.3333 & 0 \\
\hline 75 & 0.035 & 2.5 & 0 \\
\hline 80 & 0.035 & 2.6667 & 0 \\
\hline 85 & 0.035 & 2.8333 & 0 \\
\hline
\end{tabular}

\section{Conclusion}

The results show that the Windowing and EMD function proposed in this work is very good for ECG signal analysis. Not only can it get the highest SNR, but also keep the similarity and smoothness of denoised signal. In fact, this function also can be used for all kinds of signals analyze. We are analyzing R1 and R2 signal and we find out that analysis schemes involving window and EMD are able to analyze ECG signals more accurately. The improvement of signal to noise ratio for proposed method proves that this is powerful technique for analysis of non-stationary signals such as ECG signals. Now-a-days, analyses are common in communication channels and the recovery of the transmitted signals from the communication path without any noise is considered as one of the difficult tasks. This is accomplished by this analysis technique. In this paper we 
have included the newly designed matched window filter and EMD concept for better signal analysis and disturbance detection in the communication systems.

\section{References}

[1] Kabir, M.A.,Shahnaz- An ECG signal denoising method based on enhancement algorithms in EMD and Wavelet domains .IEEE conference TENCON 2011

[2] Lakhwani, R. ; Ayub, S. ; Saini, J.P. Design and Comparison of Digital Filters for Removal of Baseline Wandering from ECG Signal .IEEE Computational Intelligence and Communication Networks (CICN), 2013

[3] Bhogeshwar, Sande Seema ; Soni, M.K. ; Bansal, Dipali, Design of Simulink Model to denoise ECG signal using various IIR \& FIR filters Optimization, Reliabilty, and Information Technology IEEE(ICROIT), 2014

[4] Patil, H.T. ; Holambe, R.S. New approach of threshold estimation for denoising ECG signal using wavelet transform, IEEE (INDICON), 2013

[5] Isa, S.M. ; Noviyanto, A. ; Arymurthy, A.M.Optimal selection of wavelet thresholding algorithm for ECG signal denoising Advanced Computer Science and Information System IEEE(ICACSIS), 2011.

[6] Jenitta, J. ; Rajeswari, A. Denoising of ECG signal based on improved adaptive filter with EMD and EEMD Information \& Communication Technologies (ICT), 2013 IEEE.

[7] Kang-Ming Chang (2010). Ensemble empirical mode decomposition based ECG noise filtering method, IEEE(ICMLC).

[8] Lihuang She; Zhongqiang Xu; Shi Zhang; Yuning Song (2010). De-noisng of ECG based on EMD improved-thresholding and mathematical morphology operation, IEEE(ICBEI)2011.

[9] N. Li and P. Li, "An improved algorithm based on EMD-wavelet for ECG signal de-noising," in International Joint Conference on Computational Sciences and Optimization, 2009, pp. 825 - 827

[10] M. Steinbuch and M.J.G. van de Molengraft. Wavelet theory and applications: A literature study. Technical report, Eindhoven University of Technology, 2005

[11] Carle purdey, Harlod W. carter, Wen ben Jone - Signal Denoising Using Wavelets || CINCINNATI 2003.

[12] Moody GB, Mark RG. The impact of the MIT-BIH Arrhythmia Database. IEEE Eng in Med and Biol 20(3):45-50 (May-June 2001). 